\title{
Clinical Approach to Diagnosis of Pre-Dementia Alzheimer's Disease (CAD-PAD)
}

\author{
J. Alom ${ }^{\mathrm{a}}$ b I. Llinares ${ }^{\mathrm{b}} \quad$ S. Fajardo ${ }^{\mathrm{b}}$ \\ aMemory Clinic, Neurology Service, Hospital General Universitario Elche, Elche, and \\ ${ }^{b}$ Centro de Investigación Biomédica en Red sobre Enfermedades Neurodegenerativas \\ (CIBERNED), Spain
}

\section{Key Words}

Clinical diagnosis $\cdot$ Pre-dementia $\cdot$ Alzheimer's disease

\begin{abstract}
Objective: Our aim was to evaluate clinical and neuropsychological features to identify predementia Alzheimer's disease (PAD) among mild cognitive impairment (MCl) patients. Methods: A longitudinal prospective study of 89 consecutive patients affected with $\mathrm{MCl}$ was conducted. Clinical evaluation consisted of a clinical interview protocol, neuropsychological evaluation, standard laboratory tests, and CT scan. A 6-month clinical check-up was made to determine whether patients remained in $\mathrm{MCl}$, improved or progressed to $\mathrm{AD}$ or another dementia. Results: At 3-year follow-up, 47\% patients developed AD dementia. Seventeen variables were significant and were evaluated by logistic regression analysis to identify the remaining optimal diagnostic criteria: age, gender, repeating comments, difficulties in understanding explanations, time of symptom evolution, history of depression, and word fluency (with animals) were identified with a sensitivity of $100 \%$ and a specificity of $93 \%$. A computer application was developed with all these variables which we have named Clinical Approach to Diagnosis of PAD (CAD-PAD). Conclusions: These results suggest that CAD-PAD can help in the clinical diagnosis of PAD.




\section{Introduction}

The publication of the National Institute of Neurological and Communicative Disorders and Stroke-Alzheimer's Disease and Related Disorders Association (NINCDS-ADRDA) criteria in 1984 represented a breakthrough in the diagnosis and study of Alzheimer's disease (AD) [1]. These criteria established the clinical diagnosis of AD based on dementia with certain clinical characteristics. However, according to the experience gained in recent years, the conviction has emerged that the diagnosis of AD can in many cases be established in the predementia phase of AD. For a clinical approach, different criteria have been proposed to define the characteristics of these patients, who are commonly defined as amnestic mild cognitive impairment (MCI) patients, a term that is more widespread and more frequently used [2-4]. However, several studies that have monitored these patients for a prolonged period have revealed that approximately $50 \%$ of patients do not progress to dementia, so these criteria have not shown enough sensitivity and have not been specific enough to detect predementia AD (PAD) [5].

So, with the aim, among others, of being more certain in the diagnosis of PAD, several reviews using the NINCDS-ADRDA criteria have been conducted [6-9]. The new criteria aim to support the diagnosis with several biological markers which have shown better sensitivity and are more specific $[10,11]$. However, the role of the new criteria in daily practice remains to be defined. Cutoff points, acceptable availability and costs have not yet been established, among other issues [12].

Although the boundaries between normal cognition and MCI as well as the boundaries between nonspecific MCI and PAD are not easy to establish, we believe that the clinical history, based on a reliable informant and a neuropsychological examination (basic pillars in the evaluation of our patients), may still provide data to improve the core clinical criteria for the diagnosis of MCI due to an incipient stage of $\mathrm{AD}$. Therefore, this study was designed to evaluate whether clinical and neuropsychological characteristics could be identified in patients with MCI that could substantiate the clinical diagnosis of PAD.

\section{Methods}

A longitudinal prospective observational study of MCI patients was carried out to determine the plausible existence of clinical data with a predictive value suggestive of PAD in MCI patients.

\section{Subjects}

Consecutive MCI patients attending the Behavioral Neurology and Dementia Unit of Elche University General Hospital meeting the following inclusion criteria were recruited as cases: (1) memory impairment complaint corroborated by an informant willing to report faithfully on the patient every 6 months; (2) Clinical Dementia Rating (CDR) score of 0.5; (3) Mini-Mental State Examination (MMSE) score of $\geq 24$ ( $\geq 20$ if illiterate), and (4) complete autonomy for activities of daily living (ADL) and absence of dementia. At the same time, healthy volunteers with a trustworthy informant who could confirm that they did not suffer from memory impairment were assessed at the unit, and those who had no known neurological pathology, nor depression, and showed only a minor sensory motor disorder, if at all, were recruited as controls.

Patients and controls were submitted to clinical evaluation, consisting of an interview plus the collection of clinical data and data from neuropsychological and complementary tests. 
The monitoring period was 3 years. All patients had a follow-up visit every 6 months, where their autonomy in ADL and their neuropsychological state were re-evaluated, so that a neurologist and a neuropsychologist could reach a consensus on whether the patient remained MCI (also called MCI non-converters) or progressed to dementia (becoming a PAD patient).

All patients and controls recruited gave their informed consent. The study was developed in agreement with legal stipulations in Spain for observational epidemiologic studies and with the World Medical Association Declaration of Helsinki [13]. The study was approved by the Scientific Research Ethics Committee of Elche General University Hospital.

\section{Clinical Interview Protocol}

The following data were collected: age, gender, years and level of education, professional level, family history of dementia, sensorial/motor deficit, presence and/or history of depression [if symptoms of depression were present, the Hamilton Depression Rating Scale (HDRS) [14] was applied], presence of other diseases causing initial symptoms of cognitive decline, whether the patient came alone or was accompanied, disease course (progressive, stable, regressive, or fluctuant), time of symptom evolution, any limitation in ADL (asking about housing, shopping, cooking, finances, errands, medication, hobbies, or other usual activities), and verification of cognitive decline over the last 5 years.

Finally, questions were asked about whether the subject repeats comments or questions (spontaneously or when asked), remembers more/better remote facts than recent events (spontaneously or when asked), or shows isolated cases of (temporal and/or spatial) disorientation and difficulties in understanding explanations. In practice, the informant is asked if, in their usual contact with the patient, more detailed explanations have to be given than usual. The intention is to find out whether slight and occasional flaws exist in grasping the message of an explanation or conversation of any complexity, which could easily be compensated with a more detailed explanation and which does not interrupt the patient's ADL.

\section{Neuropsychological Assessment}

The tests performed and scales used in our patients were the CDR scale [15], the MMSE [16], several subtests of the Wechsler Memory Scale [17] revised (WMS-R) [18] and the Wechsler Adult Intelligence Scale (WAIS) [19] including the following tests: general information, orientation, mental control, logical memory, associated learning, digit span (DS) forward and reverse, digit symbol substitution, and block design. The visual memory test from 'Test de Barcelona' [20] assesses non-verbal memory: cards with geometrical figures of different sizes are shown to patients for $15 \mathrm{~s}$; thereafter, they have to recognize the figures among 4 possibilities on another card without any visual cue, obtaining 1 point for each figure recognized correctly. Other tests conducted were the Trail-Making Test [21] part A, the Boston Naming Test (BNT) [22], word fluency for animals (a) and letter P [23].

\section{Complementary Tests}

Blood analysis, TSH-T4, vitamin B12, folic acid, lues (Treponema) serology and cranial CAT scan were performed to detect and exclude possible secondary causes of MCI.

\section{Statistics}

Descriptive statistics were applied to all variables, including measures of central tendency and of statistical variability for quantitative variables, as well as absolute and relative frequencies for qualitative variables, with $95 \%$ confidence intervals for both types of variables. The Kolmogorov-Smirnov test was applied to test for the normal assumption of vari- 
ables. For those variables which did not comply with Gauss distribution, non-parametric statistics were used.

MCI non-converters were compared to PAD patients by analytical statistics. Observed differences between these two groups were analyzed by the Student $t$ test for independent data for quantitative variables and the $\chi^{2}$ test for qualitative variables. Regression analysis was performed with every variable that showed significant differences between MCI nonconverters and PAD patients at baseline.

The assessments were considered statistically significant if the two-sided $p$ value was $<0.05$. The SPSS program was used for all statistical analyses.

\section{Results}

Characteristics of Cases and Controls

Eighty-nine MCI patients and 63 controls were recruited with similar baseline demographic characteristics (table 1). Significant differences were observed in their neuropsychological analysis and in other clinical variables (table 1). The MMSE score was significantly $(\mathrm{p} \leq 0.0001)$ lower in the case $(25.8 \pm 2.7)$ than in the control group $(28.0 \pm 2.1)$, and a lower percentage of cases $(\mathrm{p}=0.002)$ reached the maximum score for the delayed memory domain.

There was a significantly higher percentage of subjects with the maximum score (better) for general information (5), orientation (5), and mental control (2-3) in the control than in the case group. Except for DS forwards, for which both groups showed similar scores, for all other Wechsler subtests, and for visual memory, BNT, and word fluency a and P, the control group showed significantly higher (better) scores. The largest difference was for the Wechsler logical memory and visual memory test, where the mean score value obtained by the cases was inferior $>1$ SD from the control value. For trail A, MCI patients showed a significantly higher (worse) ( $\mathrm{p} \leq 0.0001)$ score than the control group.

A significantly higher percentage of MCI patients than controls repeated comments/ questions, remembered more/better remote events, showed isolated cases of place disorientation, and experienced difficulties in understanding explanations.

According to inclusion criteria, controls were only selected if they did not show depression or had not had a history of it. Among MCI patients, $16 \%$ had depression and a similar percentage had a history of it.

\section{Characteristics of PAD and MCI Non-Converters}

At the 3-year monitoring of all patients, 4 patients had dropped out ( 2 had a stroke, 1 lung cancer, and 1 was missing), 45 had not progressed (MCI non-converters), and 40 developed $\mathrm{AD}$ dementia (25\% in the first, $12 \%$ in the second, and $10 \%$ in the third year), representing $47 \%$ of the MCI patients initially recruited. An analysis of clinical variables at the first visit of PAD (MCI converters) and MCI non-converter patients identified several variables for which there was a significant difference between the two groups (table 2). PAD patients included a significantly higher percentage of men $(p=0.006)$, more of them had come along with someone on their first visit $(\mathrm{p}=0.031)$, and they were significantly older $(\mathrm{p}=0.002)$ and had a shorter evolution time $(\mathrm{p}=0.016)$ than non-converters. The CDR sum of box scores was calculated as a measure of disease severity, and a significantly higher percentage of PAD patients with a sum $>0.5$ was identified ( $\mathrm{p}=0.01$ ).

Both groups had similar MMSE scores but differences in Wechsler subtests. There was a significantly lower percentage of PAD patients with the maximum score (5) for orientation $(\mathrm{p}=0.017)$, and these patients also showed significantly worse scores for logical memory 
Table 1. Demographic and clinical characteristics of MCI patients and controls

\begin{tabular}{|c|c|c|c|}
\hline & $\begin{array}{l}\text { Control } \\
(n=63)\end{array}$ & $\begin{array}{l}\text { MCI } \\
(n=89)\end{array}$ & $\mathrm{p}$ value \\
\hline Age, years & $70.2 \pm 6.9$ & $72.3 \pm 7.3$ & NS \\
\hline Female gender & 69.9 & 61.6 & NS \\
\hline Education, years & $7.1 \pm 3.5$ & $6.51 \pm 4.9$ & NS \\
\hline Family history of dementia & 33.3 & 30.8 & NS \\
\hline MMSE & $28.0 \pm 2.1$ & $25.8 \pm 2.7$ & $\leq 0.0001$ \\
\hline Delayed memory (2-3) & 91.2 & 67.2 & 0.002 \\
\hline Intrusions & 4.1 & 4.9 & NS \\
\hline \multicolumn{4}{|l|}{ Wechsler } \\
\hline General information (5) & 95.2 & 58.8 & $\leq 0.0001$ \\
\hline Orientation (5) & 100 & 78.8 & 0.002 \\
\hline Mental control (2-3) & 100 & 91.8 & 0.026 \\
\hline Logical memory & $10.6 \pm 2.5$ & $7.7 \pm 3.2$ & $\leq 0.0001^{\mathrm{c}}$ \\
\hline Associated learning & $15.9 \pm 3.7$ & $12.4 \pm 4.4$ & $\leq 0.0001$ \\
\hline DS forwards & $4.8 \pm 0.7$ & $4.6 \pm 0.8$ & NS \\
\hline DS backwards & $3.8 \pm 0.8$ & $3.3 \pm 0.8$ & 0.003 \\
\hline DSST & $19.2 \pm 7.5$ & $15.5 \pm 7.3$ & 0.007 \\
\hline Block design & $27.0 \pm 9.5$ & $21.8 \pm 10.4$ & 0.005 \\
\hline Visual memory & $8.3 \pm 1.2$ & $6.9 \pm 1.9$ & $\leq 0.0001^{\mathrm{c}}$ \\
\hline BNT & $49.3 \pm 6.3$ & $43.9 \pm 17.1$ & 0.001 \\
\hline Word fluency (a) & $19.0 \pm 4.6$ & $14.4 \pm 4.3$ & $\leq 0.0001$ \\
\hline Word fluency (P) & $13.2 \pm 4.7$ & $10.6 \pm 5.1$ & 0.003 \\
\hline Trail-Making Test part A & $73.6 \pm 33.8$ & $105.6 \pm 51.5$ & $\leq 0.0001$ \\
\hline Repeats $^{\mathrm{a}}$ & 1.6 & 71.1 & $\leq 0.0001$ \\
\hline Spontaneously & 0.0 & 31.3 & \\
\hline When asked & 1.6 & 39.8 & \\
\hline Remembers remote $^{\mathrm{b}}$ & 9.5 & 44.6 & $\leq 0.0001$ \\
\hline Spontaneously & 1.6 & 19.3 & \\
\hline When asked & 7.9 & 25.3 & \\
\hline \multicolumn{4}{|l|}{ Isolated disorientation } \\
\hline Place & 4.8 & 38.6 & $\leq 0.0001$ \\
\hline Time & 1.6 & 6.0 & NS \\
\hline DIFUNEX & 0.0 & 45.2 & $\leq 0.0001$ \\
\hline Sensory motor defect & 14.3 & 19.5 & NS \\
\hline History of depression & 0.0 & 16.3 & - \\
\hline Depression & 0.0 & 15.9 & - \\
\hline Another comorbidity & 0.0 & 29.6 & - \\
\hline
\end{tabular}

Variables are expressed as means \pm SD or as percentages. DSST = Digit symbol substitution test; word fluency $(\mathrm{a})$ = animal; word fluency $(\mathrm{P})$ = letter P; DIFUNEX = difficulty in understanding explanations; NS = non-significant.

${ }^{a}$ Repeats comments/questions. ${ }^{b}$ Remembers more/better far away events. ${ }^{c}$ The value of the case differs from that of the control by $>1 \mathrm{SD}$.

( $\mathrm{p}=0.001)$, associated learning $(\mathrm{p}=0.001)$, and block design $(\mathrm{p}=0.029)$. However, there was a significantly higher percentage of PAD patients with the maximum score (2-3) for mental control $(p=0.003)$, and they also showed a significantly better score $(p=0.01)$ for DS backwards than non-converters.

Regarding the other cognitive tests, PAD patients had a significantly $(\mathrm{p}=0.006)$ worse score for animal word fluency, and a significantly $(\mathrm{p}=0.0001)$ higher percentage of them 
Table 2. Demographic and clinical characteristics of PAD and MCI non-converter patients

\begin{tabular}{|c|c|c|c|}
\hline & $\begin{array}{l}\text { Non-converters } \\
(\mathrm{n}=45)\end{array}$ & $\begin{array}{l}\text { PAD patients } \\
(\mathrm{n}=40)\end{array}$ & $\mathrm{p}$ value \\
\hline Age, years & $69.9 \pm 7.6$ & $75.1 \pm 5.0$ & 0.002 \\
\hline Female gender & 77.8 & 45.0 & 0.002 \\
\hline Education, years & $5.3 \pm 3.2$ & $6.6 \pm 4.9$ & NS \\
\hline Low professional level & 93.4 & 70 & 0.013 \\
\hline Evolution time, months & $31.2 \pm 16.6$ & $23.3 \pm 12.3$ & 0.016 \\
\hline Reason for 1st visit: memory complaint & 92.5 & 100 & NS \\
\hline Initial course: insidious & 95.0 & 97.4 & NS \\
\hline Evolution: progressive & 80.0 & 89.5 & NS \\
\hline Comes alone & 17.5 & 2.6 & 0.031 \\
\hline CDR sum of boxes $=0.5$ & 90 & 57.5 & 0.01 \\
\hline MMSE & $25.3 \pm 2.7$ & $25.8 \pm 2.8$ & NS \\
\hline Delayed memory (2-3) & 75.0 & 68.4 & NS \\
\hline Intrusions & 12.5 & 10.5 & NS \\
\hline \multicolumn{4}{|l|}{ Wechsler } \\
\hline General information (5) & 90 & 77.8 & NS \\
\hline Orientation (5) & 97.5 & 94.5 & 0.017 \\
\hline Mental control (2-3) & 90 & 94.5 & 0.003 \\
\hline Logical memory & $8.4 \pm 2.4$ & $6.2 \pm 3.4$ & 0.001 \\
\hline Associated learning & $13.4 \pm 4.8$ & $10.1 \pm 3.1$ & 0.001 \\
\hline DS forwards & $4.3 \pm 0.8$ & $4.7 \pm 0.8$ & NS \\
\hline DS backwards & $3.1 \pm 1.0$ & $3.3 \pm 0.8$ & 0.01 \\
\hline DSST & $14.2 \pm 8.2$ & $14.7 \pm 7.4$ & NS \\
\hline Block design & $23.1 \pm 10.6$ & $17.5 \pm 8.8$ & 0.029 \\
\hline Visual memory & $6.6 \pm 1.7$ & $6.7 \pm 1.9$ & NS \\
\hline $\mathrm{BNT}$ & $44.3 \pm 6.3$ & $42.5 \pm 7.9$ & NS \\
\hline Word fluency (a) & $15.4 \pm 3.9$ & $12.8 \pm 4.2$ & 0.006 \\
\hline Word fluency (P) & $10.7 \pm 5.8$ & $9.6 \pm 4.1$ & NS \\
\hline Trail-Making Test part A & $119 \pm 51.9$ & $103 \pm 49.9$ & NS \\
\hline Repeats $^{\mathrm{a}}$ & 57.5 & 92.1 & $\leq 0.0001$ \\
\hline Spontaneously & 10.0 & 52.6 & \\
\hline When asked & 47.5 & 39.5 & \\
\hline Remembers remote ${ }^{\mathrm{b}}$ & 40.0 & 57.9 & NS \\
\hline Spontaneously & 12.5 & 31.6 & \\
\hline When asked & 27.5 & 26.3 & \\
\hline \multicolumn{4}{|l|}{ Isolated disorientation } \\
\hline Place & 40.0 & 34.2 & NS \\
\hline Time & 2.5 & 7.9 & NS \\
\hline DIFUNEX & 22.5 & 65.8 & $\leq 0.0001$ \\
\hline Minor sensory motor defect & 35.0 & 13.2 & 0.023 \\
\hline History of depression & 55.6 & 2.5 & 0.001 \\
\hline Depression & 17.5 & 13.2 & NS \\
\hline Another comorbidity & 40.0 & 21.1 & NS \\
\hline
\end{tabular}

Variables are expressed as means \pm SD or as percentages. DSST $=$ Digit symbol substitution test; word fluency $(\mathrm{a})$ = animal; word fluency $(\mathrm{P})$ = letter P; DIFUNEX = difficulty in understanding explanations; NS = non-significant.

${ }^{a}$ Repeats comments/questions. ${ }^{b}$ Remembers more/better far away events. 
Fig. 1. CAD-PAD: the selected clinical variables of the LRA and its corresponding computer application. difunex $=$ Difficulty in understanding explanations; Hdepre = history of depression; time evol $=$ time of evolution.

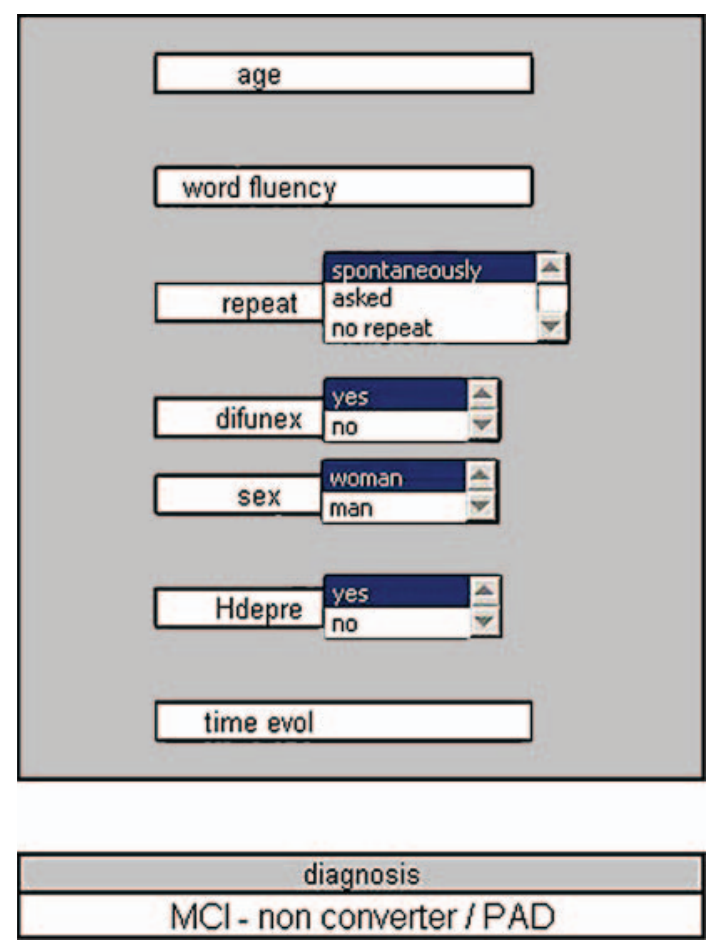

repeated comments/questions and showed difficulties in understanding explanations. On the other hand, a higher percentage of non-converters had sensory motor defects $(\mathrm{p}=0.023)$ and a history of depression $(\mathrm{p}=0.001)$.

Regression analysis was performed with the 17 significantly different variables to select the ones that best differentiated PAD patients from non-converters. The analysis was done stepwise; thus, not only was the variable selected by the algorithm, but the order by which the variable improves the significance of the overall function was selected as well. Seven variables in the following order were selected: history of depression, difficulties in understanding explanations, word fluency (a), evolution time, gender, repetition, and age. In this type of patients, the 7 mentioned variables had an overall sensitivity of $100 \%$ and a specificity of 93.2\%. A computer application was developed with all these variables to input data and obtain a score equivalent to PAD or MCI non-converter which we have named CAD-PAD (fig. 1).

\section{Discussion}

This study shows that, in MCI patients, clinical and neuropsychological features exist which allow the identification of those with PAD. In patients with PAD, 17 variables have proved to differ from those MCI who do not progress to dementia (table 2). Logistic regression analysis (LRA) of these variables selects 7 that identify patients with PAD with a sensitivity of $100 \%$ and a specificity of $93 \%$. These variables are age, gender, repetition of comments and/or questions, difficulty in understanding explanations, evolution time, history (or not) of depression, and verbal fluency (animals). Furthermore, the LRA has been elaborated by way of a computer application for diagnostic use in medical practice which we have named CAD-PAD (fig. 1). 
The inclusion criteria, using clinical and neuropsychological characteristics of patients (table 1), and the fact that $47 \%$ of patients in the $\mathrm{AD}$ series progressed to dementia within 3 years (which is comparable to findings of the majority of follow-up studies [5]) show, in essence, that the group studied is representative of patients with MCI included in many studies on the subject as well as of those patients we usually see in the daily clinic visits.

In this study, we have included a set of clinical variables in an attempt to collect data which, in clinical practice, help establish which features raise a suspicion of PAD. The clinical variables that showed significant differences were age, the tendency to repeat comments and/or questions, the difficulty in understanding explanations, a history of depression, gender, low professional level, time evolution of cognitive symptoms, going alone or accompanied to the visit, and the presence or absence of minor sensorial deficits and/or motor disorders. However, other clinical variables (remember more/better remote facts than recent events and isolated cases of temporal and/or spatial disorientation) that apparently may also suggest the beginning of PAD showed no statistical significance.

Age is the clinical variable that has most consistently had predictive significance in the literature on this subject, showing a clear increase in the incidence of AD with age [24, 25]. Our study also showed a predictive significance of age, and the variable is chosen in the LRA.

One of the variables that has proven to be the key element in the clinical profile of PAD has been the systematic collection of interviews is, according to the informant, the patients repeated comments and/or questions. In our study, with clear significance, cognitive impairment in patients with MCI who had this characteristic was highly likely to worsen in the following 3 years (included in the LRA). It is well known that this is a distinctive characteristic of $\mathrm{AD}$, but also, based on the data provided, it seems clear that many of those with PAD may show this characteristic for some time, while their MMSE scores are still high and their daily activities do not require any kind of restriction or supervision, as they are able to perform responsible tasks without any problems, for example, caring for grandchildren, banking, and administrative matters. Another clinical variable which has shown high significance when establishing the profile of PAD has been difficulties in understanding explanations (included in the LRA). These difficulties are slight and occasional flaws in receiving the message of an explanation or conversation of any complexity that can easily be compensated with a more detailed explanation and do not significantly interfere with patients' daily activities.

In our study, although the percentage of patients with symptoms of depression in the initial assessment was similar in MCI non-converters and in the PAD group, symptoms of depression were present in the patients' previous history, and much more frequently so in the MCI non-converters ( $\mathrm{p}=0.001$, included in the LRA). We believe that, although symptoms of depression at the beginning of MCI (especially if no prior history exists) must be considered in a special way, the presence of a history of depression, however distant and clearly overcome, may in some cases determine slight decreases in motivation and consequently in attitudes to daily activities, which in turn hinders the healthy cognitive stimulation. In any case, the influence of depression (of recent onset or distant) on cognitive impairment and its inclusion in the prognostic assessment of a patient with MCI is still controversial in the literature and, of course, in clinical practice [26-29].

In our study, it was found that a low-ranking job is more frequent in those with MCI who did not develop dementia, which can be explained by these patients' lower capability and/or ease in performing the neuropsychological tests leading to a misinterpretation of the test results, which indicate greater cognitive impairment and distort the overall assessment of these patients. On the contrary, and unlikely according to population-based studies, the level of education was not significantly different in MCI patients whose cognitive impairment progressed and in those where it did not. The design of the study, the origin of our patients from a memory clinic, fewer case studies compared to population studies, as well as the small in- 
fluence of this aspect on the evolution of an established MCI are facts which do not allow us to evaluate this observation.

On the other hand, the gender of the patients showed significant differences (included in the LRA). Although in the initial global MCI group the proportion of women was higher (61.6\%), 3-year monitoring showed a greater number of women (77.8\%) in the MCI nonconverter group and only $45 \%$ in the PAD group. That is to say, there is a higher predisposition in males for MCI due to PAD. The lower predisposition for PAD among women in our study required additional analyses (data not shown) which revealed that the women's group presented statistically significant differences at the first assessment compared to the male group: lower professional level, more frequent history of depression, and lower scores on the MMSE and DS reverse. The presence of these variables in the women's group (possibly influenced by socio-cultural aspects) can influence the development of a non-specific MCI which moves away from the clinical profile of PAD. In the literature, although some studies suggest a higher prevalence of MCI in men [30,31], this is a detail which is not confirmed [32].

Another clinical variable which was statistically significantly more frequent in MCI non-converters than in PAD patients was an increased presence of small sensory deficits (auditory or visual) or motor deficits (mobility defects due to osteoarthritis or other reasons), an aspect that may contribute to a decrease in daily activities with a consequent loss of cognitive stimulation and difficulties in carrying out the tests during clinical assessment. On the other hand, it was found that a greater proportion of patients who were MCI non-converters came alone to the clinic compared to PAD patients (17.5 and 2.5\%, respectively), which offers additional proof of the benignancy of the cognitive impairment of MCI nonconverters compared to PAD patients.

The evolution time of symptoms at the time of the first assessment (variable included in the LRA) was shorter in patients with PAD, which has been shown to a greater or lesser degree in other studies [25]. We believe that longer periods of evolution in an MCI patient, although they may be due to a slow onset of $\mathrm{AD}$, must make us doubt that a true PAD can be related to other non-specific factors, such as those mentioned above.

In relation to the CDR scale and the neuropsychological tests performed, the results are similar to those described in the literature [33-35]. Compared to MCI non-converters, PAD patients, at the initial evaluation (table 2), showed a higher score on the CDR sum of box areas, worse results on memory tests (logical memory and associated learning) and also on block design and orientation. On the contrary, they showed better results in mental control and DS reverse, suggesting that the MCI non-converters could have slight defects in attention compared to patients suffering from PAD. Finally, word fluency (animals) was significantly lower in those with PAD, the only neuropsychological variable which the LRA selected in its predictive analysis. We believe that the results of the memory tests, although showing clearly significant differences, were not selected in the LRA because the memory defects of patients with PAD will probably be identified by the clinical variable 'repetition of comments/questions'.

In this CAD-PAD, no doubt, the presence of a reliable informant is essential to be able to obtain this information, as well as a clinic with time and sufficient expertise to assess the reliability of said information. These requirements, although elementary, can be a major limitation if they cannot be adequately met, so we believe that these results should be corroborated by additional studies. On the other hand, the availability of the computer application will facilitate the use of CAD-PAD in clinical practice. So, based on the data of the 7 selected variables of every patient, the computer application predicts the patient's final diagnosis. It is therefore neither an invasive nor expensive tool and easy to apply in clinical practice. 
To sum up, this study shows that PAD can be identified by way of a CAD. We believe that the CAD-PAD can help in the clinical diagnosis of PAD and in subsequent decisions taken in daily clinical practice and in the selection of patients for clinical trials or other research on PAD.

\section{Acknowledgment}

This work was supported by a grant from CIBERNED, ISC-III from Spain.

\section{Disclosure Statement}

The authors have no conflicts of interest to declare.

\section{References}

-1 McKhann G, Drachman DA, Folstein M, et al: Clinical diagnosis of Alzheimer's disease: report of the NINCDS-ADRDA Work Group under the auspices of Department of Health and Human Services Task Force on Alzheimer's Disease. Neurology 1984;34:939-944.

-2 Flicker C, Ferris SH, Reisberg B: Mild cognitive impairment in the elderly: predictors of dementia. Neurology 1991;41:1006-1009.

-3 Petersen RC, Doody R, Kurz A, et al: Current concepts in mild cognitive impairment. Arch Neurol 2001;58:1985-1992.

-4 Portet F, Ousset PJ, Visser PJ, et al: Mild cognitive impairment (MCI) in medical practice: a critical review of the concept and new diagnostic procedure. Report of the MCI Working Group of the European Consortium on Alzheimer's Disease. J Neurol Neurosurg Psychiatry 2006;7:714-718.

-5 Mitchell AJ, Shiri-Feshki M: Rate of progression of mild cognitive impairment to dementia - metaanalysis of 41 robust inception cohort studies. Acta Psychiatr Scand 2009;119:252-265.

-6 Robles A, Del Ser T, Alom J, et al: Proposal of criteria for clinical diagnosis of mild cognitive impairment, dementia and Alzheimer's disease. Neurologia 2002;17:17-32.

-7 Dubois B, Feldman HH, Jacova C, et al: Research criteria for the diagnosis of Alzheimer's disease: revising the NINCDS-ADRDA criteria. Lancet Neurol 2007;6:734-746.

-8 Dubois B, Feldman HH, Jacova C, et al: Revising the definition of Alzheimer's disease: a new lexicon. Lancet Neurol 2010;9:1118-1127.

-9 Albert MS, DeKosky ST, Dickson D, et al: The diagnosis of mild cognitive impairment due to Alzheimer's disease: recommendations from National Institute on Aging-Alzheimer's Association workgroups on diagnostic guidelines for Alzheimer's disease. Alzheimers Dement 2011;7:270-279.

-10 Visser PJ, Verhey F, Knol DL, et al: Prevalence and prognostic value of CSF markers of Alzheimer's disease pathology in patients with subjective cognitive impairment or mild cognitive impairment in the DESCRIPA study: a prospective cohort study. Lancet Neurol 2009;8:619-627.

-11 Jack C, Lowe V, Senjem M, et al: PiB and structural MRI provide complementary information in imaging of Alzheimer's disease and amnestic mild cognitive impairment. Brain 2008;131:665-680.

$\checkmark 12$ Hampel H, Frank R, Broich K, et al: Biomarkers for Alzheimer's disease: academia, industry and regulatory perspectives. Nat Rev Drug Discov 2010;9:560-574.

13 World Medical Association Declaration of Helsinki: Ethical principles for medical research involving human subjects. J Postgrad Med 2002;48:206-208.

-14 Hamilton M: A rating scale for depression. J Neurol Neurosurg Psychiatry 1960;23:56-62.

- 15 Hughes CP, Berg L, Danziger WL, et al: A new clinical scale for the staging of dementia. Br J Psychiatry 1982;140:566-572.

-16 Folstein MF, Folstein SE, McHugh PR: 'Mini-mental state'. A practical method for grading the cognitive state of patients for the clinician. J Psychiatr Res 1975;12:189-198. 
-17 Wechsler D: A standardized memory scale for clinical use. J Psychol 1945;19:87-95.

18 Wechsler D: Wechsler Memory Scale-Revised (WMS-R) Logical Memory Immediate and Delayed (LMS-I and LMS-II). New York, Psychological Corporation, 1987.

19 Wechsler D: Wechsler Adult Intelligence Scale Manual. New York, Psychological Corporation, 1955.

20 Peña-Casanova J: Test Barcelona Revisado. Normalidad, Semiología y Patología Neuropsicológicas. Barcelona, Masson, 2005.

21 Reitan RM, Wolfson D: The Halstead-Reitan Neuropsychological Test Battery: Theory and Clinical Interpretation. Tucson, AZ, Neuropsychology Press, 1993.

22 Kaplan E, Goodglass H, Weintraub S: Boston Naming Test. Philadelphia, Lee and Febiger, 1983.

23 Lezak MD: Neuropsychological Assessment, ed 3. New York, Oxford University Press, 1995.

-24 Visser PJ, Kester A, Jolles J, et al: Ten-year risk of dementia in subjects with mild cognitive impairment. Neurology 2006;67:1201-1207.

-25 Fellows L, Bergman H, Wolfson C, et al: Can clinical data predict progression to dementia in amnestic mild cognitive impairment? Can J Neurol Sci 2008;35:314-322.

-26 Rozzini L, Chilovi BV, Trabucchi M, et al: Depression is unrelated to conversion to dementia in patients with mild cognitive impairment. Arch Neurol 2005;62:505, author reply 505-506.

-27 Rozzini L, Chilovi BV, Trabucchi M, et al: Predictors of progression from mild cognitive impairment to Alzheimer disease. Neurology 2008;70:735, author reply 735-736.

-28 Wilson RS, Mendes de Leon CF, Bennet DA, et al: Depressive symptoms and cognitive decline in a community population of older persons. J Neurol Neurosurg Psychiatry 2004;75:126-129.

-29 Stewart R: Depressive symptoms and cognitive decline - disentangling the effect of affect. J Neurol Neurosurg Psychiatry 2004;75:5.

-30 Petersen RC, Roberts RO, Knopman DS, et al: Prevalence of mild cognitive impairment is higher in men. The Mayo Clinic Study of Aging. Neurology 2010;75:889-897.

-31 Ganguli M, Dodge HH, Shen C, et al: Mild cognitive impairment, amnestic type: an epidemiologic study. Neurology 2004;63:115-121.

-32 Haninen T, Hallikainen M, Tuomainen S, et al: Prevalence of mild cognitive impairment: a population-based study in elderly subjects. Acta Neurol Scand 2002;106:148-154.

-33 Daly E, Zaitchik D, Copeland M, et al: Predicting conversion to Alzheimer disease using standardized clinical information. Arch Neurol 2000;57:675-680.

-34 Chen P, Ratcliff G, Belle SH, et al: Patterns of cognitive decline in presymptomatic Alzheimer disease: a prospective community study. Arch Gen Psychiatry 2001;58:853-858.

-35 Tabert MH, Manly JJ, Liu X, et al: Neuropsychological prediction of conversion to Alzheimer disease in patients with mild cognitive impairment. Arch Gen Psychiatry 2006;63:916-924. 